\title{
Guest Editorial: Special Issue on Computing Architectures for Real-Time Video/Image Analysis
}

\author{
Shao-Yi Chien • Shorin Kyo $\cdot$ Tsung-Han Tsai
}

Published online: 13 November 2009

(C) 2009 Springer Science + Business Media, LLC. Manufactured in The United States

In recent years, video and image analysis tools have been increasingly employed in many real-time applications; these include lane and car recognition for intelligent transportation systems, human object segmentation and tracking for intelligent video surveillance systems, and face detection and image indexing for digital still cameras and camcorders. To implement these analysis tools in real-time applications, new computing architectures such as reconfigurable architectures, application-specific instruction-set processors, stream processing architectures, and dedicated processing elements have been developed to handle more complex real-time content analysis. It is often necessary to integrate specially designed hardware accelerators with other processors to achieve a high processing speed. Furthermore, new algorithms that are suitable for hardware design or implementation on existing architectures play important roles in such systems. The purpose of this special issue is to report on new hardware design ideas to support these video and image analysis tools.

This special issue contains two parts. The first part describes computing platform design, including vision processors and memory sub-system design. The second part describes several design case studies of image and video analysis systems, including machine learning engines and video segmentation engines.

\footnotetext{
S.-Y. Chien $(\bowtie)$

National Taiwan University,

Taipei, Taiwan

e-mail: sychien@cc.ee.ntu.edu.tw

S. Kyo

NEC System IP Core Research Laboratories,

Kawasaki, Japan

e-mail: s-kyo@cq.jp.nec.com

T.-H. Tsai

National Central University,

Taoyuan, Taiwan

e-mail: han@ee.ncu.edu.tw
}

The first part begins with two general-purpose vision processors with a single-instruction-multiple-data (SIMD) architecture that have been developed in the industry. These two new-generation designs both feature enhanced capabilities for processing higher-level video analysis tasks with different schemes. In "IMAPCAR: A 100 GOPS In-vehicle Vision Processor Based on 128 Ring Connected 4-Way VLIW Processing Elements," Kyo and Okazaki design an in-vehicle vision processor with 128 8-bit four-way verylong-instruction-word (VLIW) RISC processing element (PE) array architecture. As compared to their previous design, IMAP-CE, the new design realizes 2.5 times better performance via the improved video I/O flexibility and data remapping structure, addition of one MAC unit per PE, and increased reliability of memory structure.

In "Xetal-II: A Low-Power Massively-Parallel Processor for Video Scene Analysis," Abbo, Kleihorst, and Schueler design a 140 GOPS image processor with a massively parallel SIMD (MP-SIMD) architecture comprising 320 PEs arranged as a linear processor array. To support regionbased processing, it provides a low-cost look-up table (LUT) and flag aggregation and flag-based result selection.

In addition to computation engines, the design of memory sub-systems also plays an important role in a video and image analysis system. In "Streaming Data Movement for Real-Time Image Analysis," López-Lagunas and Chai propose the notion of stream descriptors as a means to define image stream access patterns and to improve memory access efficiencies by discovering the locality between different data streams. Examples are provided with a Reconfigurable Streaming Vector Processor $\left(\mathrm{RSVP}^{\mathrm{TM}}\right.$ ), and the design concept can be widely applied on different computing platforms such as ASIC and reconfigurable hardware.

The second part describes four case studies that target different computing platforms: FPGA, multi-core processor, reconfigurable processor, and hybrid computing platform. 
Machine learning algorithms are widely employed in many image and video analysis applications. In "Accelerating Machine-Learning Algorithms on FPGAs Using Pattern-Based Decomposition," Nagarajan et al. propose a pattern-based decomposition design approach in which several computation patterns and communication patterns are defined, and designers can design hardware using these patterns to increase design productivity via design reusability. Some machine learning algorithms such as multi-dimensional probability density function estimation using Gaussian kernels, K-means clustering, and correlation are implemented on an FPGA as examples.

Video object segmentation is a key operation for content-based video analysis and object recognition. One popular approach is to compare the input frame with a background model. In "Real-time Adaptive Background Modeling for Multi-Core Embedded Systems," Apewokin et al. propose an adaptive background model called the multimodal mean that achieves faster execution and requires lower storage than a mixture of Gaussians. The partition of this algorithm on an embedded multi-core computing platform is also discussed.

In order to accelerate different video object segmentation algorithms, in "Reconfigurable Morphological Image Processing Accelerator for Video Object Segmentation," Chien and Chen design a reconfigurable morphological PE array that contains a reconfigurable datapath for morphology operations in each PE and a programmable interconnection unit between PEs. The configuration of this reconfigurable accelerator can be defined by a firmware for different applications. A prototype chip is also designed to show the cost efficiency of this design.

This special issue concludes with "System Level Design and Implementation for Region-of-Interest Segmentation," where Tsai et al. design a complete video object segmentation system on a board composing of an FPGA and a DSP. In this work, several system design tasks are discussed, including computationally efficient algorithm design, task partition, and system optimization techniques.

We would like to thank all the contributors of this special issue for their excellent works and the reviewers for their constructive comments, and we would also like to thank Professor Sun-Yuan Kung, Editor-in-Chief, for providing us this opportunity to collect these works in a special issue. It is expected that image and video analysis will play an increasingly important role in multimedia applications. With these representative papers in this special issue, including several processors designed by the industry and new ideas and concepts proposed by the academia, we hope to see the commencement of more related research works in the future.

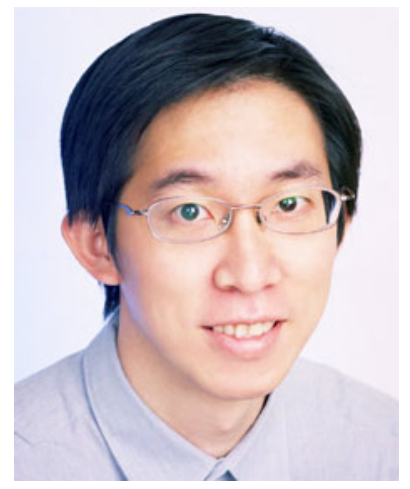

Shao-Yi Chien received the B.S. and Ph.D. degrees from the Department of Electrical Engineering, National Taiwan University (NTU), Taipei, Taiwan, in 1999 and 2003, respectively. During 2003 to 2004, he was a research staff in Quanta Research Institute, Tao Yuan County, Taiwan. In 2004, he joined the Graduate Institute of Electronics Engineering and Department of Electrical Engineering, National Taiwan University, as an Assistant Professor. Since 2008, he has been an Associate Professor. His research interests include video segmentation algorithm, intelligent video coding technology, perceptual coding technology, image processing for digital still cameras and display devices, computer graphics, and the associated VLSI and processor architectures. He has published more than 120 papers in these areas.

Dr. Chien serves as an Associate Editor for IEEE Transactions on Circuits and Systems for Video Technology and Springer Circuits, Systems and Signal Processing (CSSP). He also serves on the technical program committees of several conferences, such as ISCAS, A-SSCC, and VLSI-DAT.

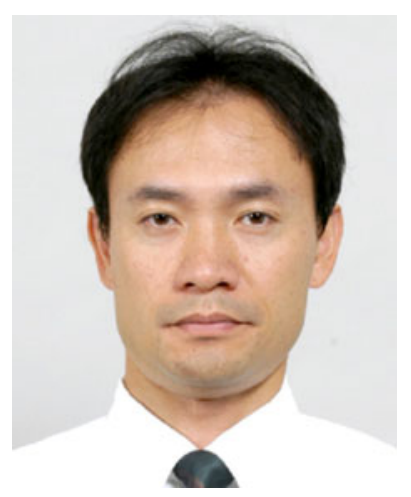

Shorin Kyo received the B.E., M.E., and Ph.D. degrees in precision engineering from the University of Tokyo in 1987, 1989, and 2004, respectively. He joined NEC Corporation in 1989, and is currently a principal researcher of System IP Core Research Laboratories. He has been involved in research on parallel processing system and processor architecture, parallel language and compiler design, and parallel image processing. In 1994-1995, he was a visiting researcher at Delft University of Technology in the Department of Applied Physics. He is a member of Information Processing Society of Japan (IPSJ), Institue of Electronics, Information and Communication Engineers (IEICE), and IEEE. 


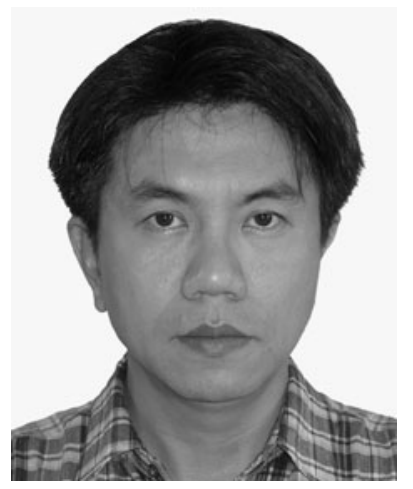

Tsung-Han Tsai received the B.S., M.S., and Ph.D. degrees in electrical engineering from the National Taiwan University, Taipei,
Taiwan, in 1990, 1994, and 1998 respectively. From 1999 to 2000, he was an Associate Professor of electronic engineering at $\mathrm{Fu}$ Jen University. He joined National Central University in 2000. Currently, he is a Professor in the department of electrical engineering at National Central University. He has been an IEEE member for over 10 years, and is also a member of the Audio Engineering Society (AES) and the Institute of Electronics, Information and Communication Engineers (IEICE). Dr. Tsai has been awarded more than 15 patents and 150 refereed papers published in international journals and conferences. His research interests include VLSI signal processing, video/audio coding algorithms, DSP architecture design, wireless communication, and System-On-Chip design. Dr. Tsai received the Industrial Cooperation Award in 2003 from the Ministry of Education, Taiwan. He is a member of the Technical Committee of IEEE Circuits and Systems Society, and serves as Technical Program Committee member or Session Chair of several international conferences. 